\title{
Impuesto sobre las emisiones de dióxido de carbono: un análisis para el caso europeo ${ }^{*}$
}

\author{
Recibido: 12 de abril de 2020 - Aprobado: 22 de mayo de 2020 \\ https://doi.org/10.22395/seec.v23n54a10 \\ David Andrés Camargo Mayorga**
Liliana Elizabeth Ruiz Acosta
Octavio Cardona García ${ }^{* * *}$
}

\section{RESUMEN}

El presente artículo realiza un análisis de datos panel con vectores autorregresivos (PVAR) para determinar la efectividad de los impuestos ambientales sobre la emisión de dióxido de carbono $\left(\mathrm{CO}_{2}\right)$ en los sectores de minería y transporte en treinta y un países europeos. Se hace el análisis econométrico con variables simultáneas, como lo son los impuestos y la generación de $\mathrm{CO}_{2}$, las cuales se afectan una con la otra. El resultado más destacado de este estudio muestra que las emisiones de $\mathrm{CO}_{2}$ de los sectores de transporte y minería no se ven afectadas por los impuestos. Esta discusión es importante para Colombia, que recién cobra impuestos al carbono y tiene potencial en el uso de energías fósiles que generan emisiones contaminantes.

\section{PALABRAS CLAVE}

Tributación; contaminación; minería; transporte; energía.

\section{CLASIFICACION JEL}

$\mathrm{C} 32, \mathrm{C} 33, \mathrm{H} 21, \mathrm{H} 23$

\section{CONTENIDO}

Introducción; 1. Revisión de literatura; 2. Metodología; 3. Resultados y discusión; Conclusiones; Bibliografía.

Este artículo es un producto del proyecto de investigación titulado: Instrumentos tributarios de la política y gestión ambiental colombiana financiado por la Universidad Central en la vigencia 2017.

* Economista y Magíster en Economía, Pontificia Universidad Javeriana, Bogotá, Colombia. Magister en Educación, Universidad Militar Nueva Granada, Bogotá, Colombia. Docente Investigador de tiempo completo y miembro del grupo de Estudios Contemporáneos en Contabilidad, Gestión y Organizaciones, Facultad de Ciencias Económicas, Universidad Militar Nueva Granada, Bogotá, Colombia. Correo electrónico: david.camargo@ unimilitar.edu.co. Orcid: https://orcid.org/0000-0002-5290-8251.

*. Contador Público, Universidad Mariana, Pasto, Colombia. Magíster en Gestión Empresarial de la Universidad Libre, Cali, Colombia. Docente y miembro del grupo Ataralwa-Ama, Departamento de Contaduría Pública, Universidad Central, Bogotá, Colombia. Docente Investigador de tiempo completo y miembro del grupo GECS, Facultad de Ciencias Económicas, Universidad Militar Nueva Granada, Bogotá, Colombia. Correo electrónico: liliana.ruiz@unimilitar.edu.co. Orcid: https://orcid.org/0000-0003-3323-8480.

.... Economista, Universidad Militar Nueva Granada, Bogotá, Colombia. Miembro del grupo de Estudios Contemporáneos en Contabilidad, Gestión y Organizaciones, Facultad de Ciencias Económicas, Universidad Militar Nueva Granada, Bogotá, Correo electrónico: octavio.cardona.g@gmail.com. Orcid: https://orcid.org/00000001-6257-5270. 


\title{
TAX ON THE CARBON DIOXIDE EMISSIONS: AN ANALYSIS OF THE EUROPEAN CASE
}

\begin{abstract}
This article performs data analysis with panel-vector autoregressive (PVAR) data for determining the effectiveness of the environmental taxes upon the emission of carbon dioxide (CO2) in the mining and transport sector in thirty-five European countries. An econometric analysis is performed with simultaneous variables as are the taxes and the $\mathrm{CO} 2$ generation, which are affecting one another The most relevant result of this study displays how the $\mathrm{CO} 2$ emissions of the transport and mining sectors are not affected by the taxes. This is a very important discussion for Colombia given that only recently it taxes carbon emissions and it has a great use potential in the fossil energy industries, which also generates contaminating emissions.
\end{abstract}

\section{KEYWORDS}

Taxation; contamination; mining; transport; energy.

\section{JEL CLASSIFICATION}

C32, C33, H21, H23.

\section{CONTENT}

Introduction; 1. Literature review; 2. Methodology; 3. Results and discussion; Conclusions; Bibliography.

\section{IMPOSTO SOBRE AS EMISSÕES DE DIÓXIDO DE CARBONO: UMA ANÁLISE PARA 0 CASO EUROPEU}

\section{RESUMO}

Este artigo realiza uma análise de painel de dados com vetores autorregressivos para determinar a efetividade dos impostos ambientais sobre a emissão de dióxido de carbono (CO2) nos setores de mineração e de transporte em 31 países europeus. Realiza-se análise econométrica com variáveis simultâneas, como ocorre com os impostos e a geração de $\mathrm{CO} 2$, as quais são afetadas uma com a outra. O resultado mais destacado deste estudo mostra que as emissões de $\mathrm{CO} 2$ dos setores de transporte e mineração não são afetadas pelos impostos. Essa discussão é importante para a Colômbia, que começou a cobrança de impostos do carbono recentemente e tem potencial no uso de energias fósseis que geram emissões contaminantes.

\section{PALAVRAS-CHAVE}

Tributação; poluição; mineração; transporte; energia.

\section{CLASSIFICAÇÃO JEL}

$\mathrm{C} 32, \mathrm{C} 33, \mathrm{H} 21, \mathrm{H} 23$

\section{CONTEÚDO}

Introdução; 1. Revisão de literatura; 2. Metodologia; 3. Resultados e discussão; Conclusões; Bibliografia. 


\section{INTRODUCCIÓN}

Los impuestos son uno de los múltiples instrumentos económicos (o "de mercado") que existen para la regulación ambiental. Según Tietenberg (1998) ha habido tres olas de regulación ambiental: la primera, de carácter legal y basada en estándares, la segunda, basada en instrumentos económicos (impuestos/subsidios, permisos transables, depósito/reembolso, sistemas de responsabilidad legal y compensación) y la tercera, basada en información (obligatoria o voluntaria) sobre contaminación.

Para Yábar (2016), dentro de las bondades que tienen los instrumentos económicos, se tienen que modificar las conductas dañosas para el ambiente, propiciar el uso eficiente de los recursos escasos, promover la innovación tecnológica (y el uso de tecnologías limpias), aplicar el principio de quien contamina, paga, para fijar precios más acordes con los costos sociales de un producto, generar más y mejores normas y producir más recaudo tributario. Los impuestos, denominados también como tributos extrafiscales, se han usado para solucionar el problema de la emisión de dióxido de carbono $\left(\mathrm{CO}_{2}\right)$ a la atmósfera (de gases efecto invernadero y de otros más). Para Yábar (2016) la modificación de los precios relativos de los bienes y servicios cambian la conducta de los productores y consumidores que contaminan. Estos impuestos gravan a los agentes económicos por una determinada cantidad de emisiones contaminantes, de manera que la externalidad' negativa se intenta corregir.

Aunque en la teoría este tipo de tributos incentivarían a los agentes a reducir su generación de contaminantes, en la realidad, aplicarlos de forma eficiente no es fácil. En este sentido, algunos de los problemas a los que se enfrentan los hacedores de política son: i) fijar los impuestos menos distorsivos, ii) no gravar a agentes clave dentro de la economía que puedan deteriorar el desempeño económico general, aunque eso dependa de la adaptación de estos al impuesto y a la compensación que hagan actividades económicas no perjudicadas, por ejemplo, la petrolera y la de energías renovables, para evitar deterioros generales de la economía, iii) no utilizar el recaudo de impuestos para corregir la externalidad. Frente a estas problemáticas, el objetivo de este artículo es analizar la efectividad de los impuestos en treinta y un países de Europa para dos sectores específicos: minería y transporte por carretera. El primero de estos, en donde la emisión de $\mathrm{CO}_{2}$ no es intensiva, pero que permite comparar los resultados con el segundo sector objeto de análisis, el de transporte por

\footnotetext{
Las externalidades son para Martínez-Alier y Roca-Jusmet (2013, p. 132) un "fallo del mercado" y se dan en la producción y el consumo. Las primeras se dan cuando las decisiones de un empresario inciden en la producción de otra empresa, mientras que las segundas se dan porque a un consumidor lo afectan la producción o el consumo que otros hacen. Para Varian (2006), en cualquier caso, estas tienen como característica principal que los bienes que las originan no tienen un mercado organizado donde comprarse y venderse.
} 
carretera. Esto, en razón a que se trata de dos sectores regulados y con datos disponibles y en la cantidad suficiente para los análisis econométricos.

La metodología utilizada para efectos del análisis es la de datos panel con vectores autorregresivos o también conocida como panel datos-VAR (PVAR). Este tipo de análisis econométricos son pertinentes, porque permiten trabajar con variables simultáneas y endógenas. Para el caso de los impuestos, se asume que pueden incidir en la generación de $\mathrm{CO}_{2}$, pero al mismo tiempo, verse afectados por la cantidad de gases contaminantes que emiten los diferentes agentes implicados, por lo tanto, son variables que pueden resultar simultáneas. Para cumplir con el objetivo propuesto, el documento se organiza de la siguiente manera: una introducción al tema, una revisión de literatura sobre impuestos a las emisiones de $\mathrm{CO}_{2}$, la descripción de la metodología empleada, los resultados y discusión de las estimaciones realizadas y las conclusiones.

\section{REVISIÓN DE LITERATURA}

La literatura que se presenta a continuación se apoya, ante todo, en trabajos que analizaron los efectos de las medidas impositivas sobre las diferentes industrias para disminuir la contaminación y mitigar el daño ambiental. Según la U.S. Energy Information Administration (2016), las políticas fiscales en términos ambientales se estructuran con base en diferentes instrumentos, se destacan los de control y los económicos; y las políticas relacionadas con el consumo de energía en el sector transporte se relacionan con el ahorro de combustibles y la política de emisión de gases de efecto invernadero.

Los impuestos, como mecanismo para reducir la contaminación, aparecen en la literatura económica para contrarrestar las externalidades negativas, que deben ser internalizadas, ya que estas suponen un costo para la sociedad. Dicho análisis es desarrollado por Pigou (1920), quien manifiesta que los impuestos sirven para incentivar a los agentes implicados a contaminar a un nivel aceptado por la sociedad. En suma, de lo anterior, los impuestos son considerados como un mecanismo eficiente y barato de control de acuerdo con autores como Baumol y Oates (1988), Barde (2002) y la OCDE (2013). Estos también crean una ganancia neta para la sociedad en el largo plazo, debido a que proporcionan incentivos para reducir las emisiones de forma directa y alientan la búsqueda de energías alternativas como lo plantea Helm (2012). Para Ito (2017), los impuestos promueven la entrada de fuentes de energía menos contaminantes en el mercado, por ejemplo, energías hidroeléctrica y eólica, combustibles de biomasa, carbón y gas natural con captura y almacenamiento de $\mathrm{CO}_{2}$, y reducen la demanda final de esta a través de mejoras 
en la eficiencia energética. De ahí que para Calderón et al. (2016) es correcto afirmar que el sector de la energía eléctrica juega un papel fundamental en la reducción de emisiones de $\mathrm{CO}_{2}$.

En general, un impuesto puede clasificarse como instrumento de gestión ambiental, si su finalidad es la de captar ingresos para la protección y conservación del ambiente, al tiempo que atenúa o previene la conducta para la cual fue creado. No obstante, la aplicación real de este instrumento económico resulta mucho más difícil, pues no es fácil determinar cuál es el nivel de carga impositiva que debe aplicarse con el menor efecto distorsivo, por lo que alcanzar un óptimo social resulta improbable. A propósito, Parry y Small (2005) comparan las medidas impositivas sobre la gasolina en Estados Unidos y Gran Bretaña, para concluir que para el año en que se realizó el estudio, el nivel óptimo de impuestos en Norteamerica estaba por debajo, ya que debía ser de más del doble, mientras que en Reino Unido estaba sobrevaluado, porque debía estar por debajo de la mitad.

Finlandia, como lo mencionan Andersen y Ekins (2010) al inicio de la década de los noventa fue el primer país que introdujo un impuesto sobre el $\mathrm{CO}_{2}$, lo que más tarde replicaron Suecia, Dinamarca, Países Bajos, Eslovenia, Alemania y el Reino Unido. En el caso de Dinamarca, para finales del siglo XX, Buhl Pedersen et al. (1998) encontraron que los tributos habían generado una reducción del $5 \%$ en la emisión de $\mathrm{CO}_{2}$ entre 1996 y 1997, y Bjørner y Jensen (2002) que estos produjeron una disminución en el consumo energético desde 1993 hasta 1997 del 10 \%. Más reciente es el estudio de Wier et al. (2005) para este mismo país, que muestra que los impuestos ambientales funcionan para reducir las emisiones de $\mathrm{CO}_{2}$, pero que, al dirigirse al consumo de energía en los hogares y la industria, han tenido un efecto regresivo que ha alterado la distribución del ingreso.

En líneas generales, Zhang et al. (2016) en un estudio bibliométrico sobre la investigación que se ha realizado en impuestos al carbono, encuentran que los estudios analizados entre 1989 y 2004 se concentran en un $43 \%$ en veinte revistas top en el tema; con trabajos interdisciplinares cuyas categorías temáticas fueron tres: combustibles, estudios ambientales y ciencias ambientales. En el análisis de las referencias encontraron que la referencia más antigua con relación al impuesto al carbono fue la de Pigou (1920) y que las palabras clave que con más frecuencia se repiten habían sido: cambio climático y política, comercio de emisiones de carbono, efectos sociales y económicos de los impuestos al carbono, energías renovables, cambio tecnológico endógeno, y captura y almacenamiento de carbono. Se rastreó que en la literatura, al menos seis tipos de modelos analíticos se usaron para indagar sobre los efectos que tienen los impuestos ambientales sobre las emisiones de $\mathrm{CO}_{2}$. 
El primero de estos es el modelo de equilibrio general. Aplicado para Noruega por parte de Bruvoll y Larsen (2004), quienes encontraron que los efectos de los impuestos sobre las emisiones son mínimos, estos apenas contribuyeron con 1,5\% de una disminución total del 2,3 \%, en un periodo de nueve años (1990-1999). Este efecto tan pequeño responde en esencia a las excepciones fiscales y a la inelasticidad precio de la demanda que existe en los sectores afectados por la carga fiscal. En el caso de China, Liang, Fan y Wei (2007) hallaron que el efecto de un impuesto sobre el carbono resultaría negativo para la economía, y sugirieron que este efecto se podría alivianar con un subsidio a los sectores de la producción. Plantean además, como alternativa de política, combinar la exención de impuestos para los sectores intensivos en energía y comercio con reembolsos de los ingresos fiscales a los sectores no exentos. Por último, para Irlanda, Wissema y Dellink (2007), después de cuantificar el impacto que tienen los impuestos a la energía sobre la generación de $\mathrm{CO}_{2}$, el principal hallazgo que hicieron fue que con un impuesto al carbón de entre 10 a 15 euros por tonelada se lograría una disminución en las emisiones del $25 \%$, teniendo como referencia el periodo de los años 1998-2006.

El modelo de equilibrio parcial es el segundo que se usó. Este fue aplicado por Nakata y Lamont (2001), que para el caso japonés concluyen que los impuestos al carbono o a la energía pueden ser efectivos, e incluso generar buenos rendimientos para el fisco. Pero este efecto se ve contrarrestado por la eliminación gradual del uso del carbón, algo que a la larga hará más dependiente a ese país de los precios internacionales del gas y el petróleo. En esta misma línea de trabajo, Calderón et al. (2016) muestran que las tecnologías de generación de electricidad afectan las emisiones de carbono, y un impuesto tiene el potencial reducir la generación de $\mathrm{CO}_{2}$. Este gravamen, según los hallazgos de estos autores, podría afectar de forma negativa el PIB del país entre $2 \%$ y $3 \%$. Además, las políticas que propician el uso de energías limpias tienden a reducir el consumo de energía, las emisiones y aumentan la eficiencia productiva.

Como tercer modelo está el de demanda de energía. Los hallazgos de Gerlagh y Van der Zwaan (2006), con la aplicación de este, muestran que al realizar el análisis de diferentes portafolios como opciones para mitigar los causantes del cambio climático, dentro de los cuales se incluyó el uso de impuestos, se concluye que el uso de los impuestos en forma de subsidios para las fuentes de energía no basadas en carbón, resulta ser la forma más barata y eficiente de combatir la problemática ambiental. Por su parte, Di Cosmo y Hyland (2013) encontraron a la luz del mismo modelo, que si Irlanda genera un cambio en los impuestos desde 21,50 euros/ tonelada de $\mathrm{CO}_{2}$ para tener como base el año 2012, a 41 euros/tonelada de $\mathrm{CO}_{2}$ en el año 2025, se reflejaría una reducción de la polución de unas 861.000 toneladas, 
en comparación con un escenario sin impuestos. De forma análoga, el efecto negativo de esta hipotética política impositiva en la macroeconomía del país se vería reflejada con una contracción del 0,21 \% en el PIB y 0,08 \% en el nivel de empleo.

Como cuarto modelo está el de crecimiento endógeno basado en la acumulación de capital humano. Al respecto, Oueslati (2014) plantea que la magnitud de los efectos sobre el crecimiento de diferentes reformas fiscales ambientales sugieren que estos dependen del tipo de reforma de impuestos y del costo de ajuste de la inversión. Aunque los ingresos del impuesto ambiental mejoran el crecimiento, sus efectos a largo plazo en el bienestar dependen del costo de ajuste del capital. Por su parte, en el corto plazo, sin importar cuál sea el escenario de la reforma de impuestos, el efecto sobre bienestar es siempre negativo.

El quinto es el modelo bayesiano estructural de series de tiempo, que usaron González y Hosoda (2016), quienes encontraron que el impuesto al combustible redujo las emisiones de $\mathrm{CO}_{2}$ a la atmósfera hechas por los vuelos domésticos en Japón.

El sexto de estos modelos fue el de optimización determinística, para el cual Wang et al. (2018), muestran que para la provincia de Hebei (China) las políticas fiscales ambientales mejoraron la calidad del aire y promovieron energías renovables.

Ahora bien, el presente documento tiene dos diferencias esenciales en comparación con los modelos antes listados. La primera, es la de reconocer que existe una relación entre impuestos y emisiones a la atmósfera que es endógena, en contravía de los modelos de equilibrio general encontrados, los cuales parten de supuestos acerca del estado del mercado, y buscan encontrar el vector de precios que se ajuste mejor a dicho mercado. De ahí que se pretenda en este artículo encontrar la relación causal entre las dos variables con una metodología que combina los datos panel y los vectores autorregresivos. Segundo, los análisis realizados en otros documentos previos suelen enfocarse en un solo país. No obstante, el problema de las emisiones tiene implicaciones regionales y globales, por lo tanto, analizar un bloque de países europeos puede dar una idea más completa de lo que ocurre con la eficiencia de la carga impositiva al carbono.

\section{METODOLOGÍA}

Los datos utilizados para desarrollar el modelo econométrico fueron obtenidos de Eurostat y de Passport-Euromonitor, y corresponden a un panel de datos desbalanceado para treinta y un países de Europa en el periodo 2004-2014. Las variables consideradas para el análisis fueron: Emisión de $\mathrm{CO}_{2}$ para la industria de minas (expresado en toneladas); impuestos ambientales totales (expresados en 
millones de euros); inversiones totales en medio ambiente para el sector de minería (expresado en millones de euros); actividades de protección ambiental para el sector de minería (expresado en millones de euros); emisión de $\mathrm{CO}_{2}$ para el sector transporte (expresado en toneladas); impuestos ambientales del sector de transporte (expresado en millones de euros) y consumo final de energía en el sector transporte (expresado en millones de toneladas de petróleo) $)^{2}$. Cada una de las variables y sus medias son presentadas en la tabla 1 para el sector minero, en donde se observa que los países con más altas emisiones en promedio fueron Reino Unido, Noruega, Alemania y Republica Checa, y los que más recaudo tributario promedio tuvieron fueron Alemania, Reino Unido, Francia e Italia. En general, los valores son disímiles entre países, de donde se resaltan diferencias como las que muestran Bélgica y Austria, que tienen casi la misma recaudación por impuestos ambientales, pero las emisiones de Austria son cuarenta y dos veces las de Bélgica.

Tabla 1. Media de las variables por países sector minero

\begin{tabular}{ccccc}
\hline País & Emisión de $\mathrm{CO}_{2}$ & Impuestos ambientales totales & $\begin{array}{c}\text { Inversión total en } \\
\text { ambiente }\end{array}$ & $\begin{array}{c}\text { Actividades de } \\
\text { protección ambiental }\end{array}$ \\
\hline Austria & 1.143 .701 & $7.191,984$ & 1,2100 & 3,9056 \\
Bélgica & 27.324 & $7.127,589$ & 0,2575 & 0,7488 \\
Bulgaria & 234.873 & $1.078,997$ & 0,5650 & 2,8300 \\
Croacia & 931.910 & $1.513,188$ & 0,5930 & 1,6070 \\
Chipre & 24.937 & 529,1278 & 0,5400 & 3,3022 \\
República Checa & 4.252 .181 & $3.432,381$ & 4,1260 & 13,4990 \\
Dinamarca & 1.772 .294 & $10.231,47$ & - & - \\
Estonia & 98.802 & 426,9589 & 0,5314 & 2,5900 \\
Finlandia & 413.091 & $5.380,889$ & 4,9311 & 11,2867 \\
Francia & 1.258 .862 & $38.911,22$ & 0,4071 & 0,4850 \\
Alemania & 4.907 .074 & $56.555,56$ & 0,7829 & 3,3343 \\
Grecia & 59.123 & $5.418,444$ & - & - \\
Hungría & 523.251 & $2.674,384$ & 0,2733 & 0,3133 \\
Irlanda & 273.045 & $4.330,646$ & - & - \\
Italia & 2.172 .627 & $48.722,22$ & 7,6463 & 13,5050 \\
Letonia & 35.955 & 484,5467 & 0,0290 & 0,1430 \\
Lituania & 9.221 & 533,0411 & 0,0300 & 0,3160 \\
Luxemburgo & 4.543 & 973,1344 & - & - \\
Malta & 4.858 & 203,8322 & 0,2850 & - \\
Holanda & 1.856 .234 & $21.698,67$ & 2,7925 & 7,9375 \\
\hline Un acercamiento al análisis para el total de las industrias se presenta en la tabla 4, en donde se calculan las \\
correlaciones por país entre los impuestos al carbón, las inversiones en medio ambiente, las actividades de \\
protección ambiental y los impuestos. Para aquellos países con menos datos faltantes se encuentra una fuerte \\
correlación negativa entre la variable de los impuestos y la generación de co \\
hubo evidencia que para el conjunto de industrias los efectos de los impuestos fueron contraproducentes. \\
\end{tabular}


Impuesto sobre las emisiones de dióxido de carbono: un análisis para el caso europeo

\begin{tabular}{ccccc}
\hline País & Emisión de $\mathrm{CO}_{2}$ & Impuestos ambientales totales & $\begin{array}{c}\text { Inversión total en } \\
\text { ambiente }\end{array}$ & $\begin{array}{c}\text { Actividades de } \\
\text { protección ambiental }\end{array}$ \\
\hline Noruega & $13.931 .480,67$ & $8.597,714$ & 34,9633 & 62,9989 \\
Polonia & 1.627 .144 & $9.264,397$ & 0,6390 & 8,2760 \\
Portugal & 299.633 & $4.214,572$ & 0,5180 & 1,0010 \\
Rumania & 1.226 .662 & $2.610,78$ & 3,6340 & 7,6910 \\
Serbia & 2.103 .332 & 965,4111 & 0,4688 & 0,8175 \\
Eslovaquia & $661.208,6$ & $1.144,833$ & 0,0844 & 0,3422 \\
Eslovenia & $87.076,29$ & $1.155,671$ & 1,7556 & 2,6544 \\
España & 3.294 .709 & 18.095 & 0,8067 & 1,3089 \\
Suecia & $929.185,4$ & $9.375,378$ & 4,1313 & 6,5700 \\
Turquía & 1.891 .530 & $23.249,32$ & 0,0575 & 0,2100 \\
Reino Unido & $19.044 .177,67$ & $48.189,61$ & 1,9289 & 4,8300 \\
\hline
\end{tabular}

Fuente: elaboración propia.

Por su parte, la tabla 2 muestra la información del valor medio de las variables para el sector transporte por carretera para cada país. En esta se identifica que en promedio los países que más emisiones tuvieron fueron Alemania, Reino Unido, Italia y Francia, y los que más recaudo promedio de impuestos mostraron fueron Reino Unido, Alemania, Italia y Holanda. Se observa que países como Serbia y Croacia tuvieron en promedio un consumo final de energía relativamente alto respecto de sus emisiones en comparación con los demás países.

Tabla 2. Media de las variables por países sector de transporte por carretera

\begin{tabular}{cccc}
\hline País & Emisión de $\mathrm{CO}_{2}$ & Consumo final de energía & Impuestos ambientales al transporte \\
\hline Austria & $6.422 .620,1$ & 8,1636364 & $2.400,1744$ \\
Bélgica & $9.286 .146,9$ & 8,7545455 & $2.689,7111$ \\
Bulgaria & $4.304 .089,6$ & 2,7545455 & 99,854444 \\
Croacia & $1.013 .670,1$ & 1,9727273 & 402,78222 \\
Chipre & $576.044,29$ & 0,69090909 & 180,62 \\
República Checa & $8.689 .110,1$ & 5,9454545 & 219,29667 \\
Dinamarca & 43.804 .506 & 4,3545455 & $3.938,6133$ \\
Estonia & $1.647 .731,7$ & 0,74545455 & 9,2244444 \\
Finlandia & 10.498 .305 & 4,2636364 & $1.733,6667$ \\
Francia & 46.269 .328 & 44,109091 & $5.627,4444$ \\
Alemania & 81.467 .586 & 54,3 & 9.010 \\
Grecia & $7.716 .603,3$ & 6,9727273 & $1.503,7778$ \\
Hungría & $4.142 .582,4$ & 4,0636364 & 511,29 \\
Irlanda & $2.850 .984,7$ & 4,0272727 & $1.790,0767$ \\
Italia & 47.130 .535 & 39,509091 & $6.317,2222$ \\
Letonia & 2.510 .600 & 1,0454545 & 63,47111
\end{tabular}


David Andrés Camargo Mayorga, Liliana Elizabeth Ruiz Acosta y Octavio Cardona García

\begin{tabular}{cccc}
\hline País & Emisión de $\mathrm{CO}_{2}$ & Consumo final de energía & Impuestos ambientales al transporte \\
\hline Lituania & $4.098 .754,1$ & 1,5181818 & 17,603333 \\
Luxemburgo & $3.470 .751,9$ & 2,2181818 & 63,75 \\
Malta & $2.755 .565,8$ & 0,16363636 & 91,5 \\
Holanda & 26.012 .981 & 11,5 & $7.003,4444$ \\
Noruega & 17.930 .150 & 4,6727273 & $3.548,96$ \\
Polonia & 22.361 .627 & 14,627273 & 755,32111 \\
Portugal & $6.050 .444,4$ & 6,1363636 & $1.102,6378$ \\
Rumania & $5.439 .217,3$ & 4,8545455 & 363,09222 \\
Serbia & $796.602,5$ & 2,0818182 & 97,883333 \\
Eslovaquia & 4.606 .101 & 2,3727273 & 120,91 \\
Eslovenia & $4.133 .392,3$ & 1,7272727 & 156,63556 \\
España & 31.736 .247 & 33,709091 & $2.786,3333$ \\
Suecia & 12.907 .922 & 7,7727273 & $1.656,0544$ \\
Turquía & 18.734 .973 & 15,563636 & $6.816,135$ \\
Reino Unido & 74.502 .069 & 41,145455 & $10.493,722$
\end{tabular}

Fuente: elaboración propia.

Antes de continuar con la descripción del modelo, es necesario explicar que los dos sectores seleccionados y sus respectivas variables responden a dos condiciones. El primer condicionante es que sobre ambos sectores hay bastante regulación en Europa, y por consiguiente, hay una mayor cantidad de datos disponibles, lo que permitió construir una base con suficientes observaciones para desarrollar el modelo econométrico. En el mismo sentido, el segundo condicionante es una restricción en la cantidad y la calidad de los datos. Como se ha mencionado con anterioridad, los reportes de los países tienen muchos vacíos, en parte porque cada país europeo fija sus reglas impositivas así pertenezcan o no a la Unión Europea, y porque hasta el año 2012 cada país proveía información cada dos años.

En ese sentido, ambos limitantes restringen el análisis, porque la variable latente "impuesto al carbono", se representó con los impuestos ambientales totales para el caso del sector minero, y los impuestos ambientales del sector de transporte. Por ende, el modelo se construyó como una aproximación de la variable no observable. El uso de la variable aproximada no se explica porque los impuestos a las emisiones de carbono no estén disponibles en reportes o bases de datos de cada uno de los países, sino más bien porque los reportes difieren por razones políticas y generan cambios exógenos que no pueden ser capturados por el modelo, además la carga impositiva no es directa como pasa en países como Chipre o algunos otros de Europa Oriental, en donde los pagos por derechos de conducción de vehículos incluyen sobrecostos (compra, matrícula o circulación), y funcionan como impuesto sobre las emisiones. En ese sentido, Hayashi, Kato y Teodoro (2001) y Ryan, Ferreira 
y Convery (2009) señalan que el impuesto de circulación anual es más influyente en la eficiencia del uso del combustible para la reducción de emisiones de $\mathrm{CO}_{2}$. Además, al analizar el problema desde una perspectiva más técnica, las variables impuestos ambientales totales e impuestos ambientales del sector transporte, por definición, están correlacionadas con los impuestos al $\mathrm{CO}_{2}$, pero no están correlacionadas con los factores capturados por el error de la regresión.

Aunque se trató de obtener una base más completa, los datos no disponibles fueron extrapolados de las observaciones ya existentes en el panel, mediante el método de extrapolación lineal. En definitiva, el modelo planteado intenta responder a la hipótesis de que el sistema de impuestos puede resultar endógeno o simultáneo, es decir, que no se puede establecer con claridad si los impuestos dependen de la contaminación generada o, al contrario; es porque es la tasa impositiva y las emisiones (no referidas a $\mathrm{CO}_{2}$, sino a cualquier otro contaminante, en general, gases de efecto invernadero). De forma alternativa, esto puede ocurrir porque existe una posible influencia de regulaciones desde el parlamento europeo, los movimientos ambientales y la postura de los stakeholders que presionan a las diferentes empresas para que contaminen menos.

Ante la incapacidad de identificar cuáles variables son exógenas al modelo, la metodología propuesta es la de datos panel con vectores autorregresivos (PVAR), planteada por Cagala et al. (2015) y programada para el software econométrico Stata 12.1 por Cagala y Glogowsky (2014). La ventaja de este modelo sobre otros en la literatura consultada es que se pueden relajar algunos supuestos y no es necesario partir desde una condición de equilibrio, sumado a que el modelo permite la comparación entre países en diferentes momentos del tiempo, y permite capturar los efectos de la carga impositiva sobre las demás variables en periodos subsecuentes y viceversa.

Dado que el modelo VAR considera todas las variables como simultáneas, no se expresa ninguna forma funcional específica, sino que para el caso de cada industria se plantean las ecuaciones 1 y 2 con los vectores que se estiman por separado.

$$
\begin{aligned}
& \mathrm{Y}_{\text {Mineria }}=\left|\begin{array}{c}
\text { Generación de dióxido de carbono } \\
\text { Impuestos ambientales totales } \\
\text { it } \\
\text { Inversiones totales en medio ambiente }_{i t} \\
\text { Actividades de protección ambiental }
\end{array}\right| \\
& \mathrm{Y}_{\text {Transporte }}=\left|\begin{array}{c}
\text { Generación de dióxido de carbono } \\
\text { Impuestos ambientales al transporte } \\
\text { Consumo total de energía }{ }_{i t}
\end{array}\right|
\end{aligned}
$$


Aunque se puede asumir que la inversión en medio ambiente y las actividades ambientales en la industria dependen en parte de los impuestos, es difícil imaginar que el caso contrario ocurra. Pero, aun así, estas actividades sí pueden responder a la generación de agentes contaminantes, y al mismo tiempo, estos dependerán de las variaciones de dichas inversiones y actividades, por tal razón se integran dentro del primer modelo (vector $Y_{\text {minería }}$ ) como variables endógenas juntos con las demás.

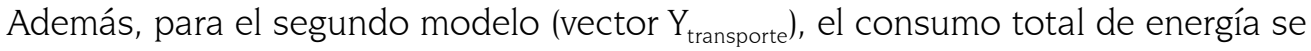
incluye como una variable relacionada con la eficiencia en el consumo de combustibles y las emisiones, según lo refiere la literatura que se revisó. Para ambos modelos las variables están expresadas en niveles y se utilizaron dos rezagos en la estimación. Tras conocer los limitantes que se mencionan en párrafos anteriores, no fue posible ejecutar ningún tipo de análisis de raíz unitaria o estabilidad de los parámetros, y para evitar errores de cómputo no se realizaron transformaciones de las variables.

Las herramientas de análisis para los dos modelos fueron las funciones impulsorespuesta, las cuales permitieron identificar los efectos que cada una de las variables tiene sobre las demás dentro del sistema de ecuaciones. Se utilizó la herramienta FEVD (Forecast Error Variance Decompositions), que sirve para conocer la fracción de la varianza de la variable dependiente, que es explicada por las demás variables en un horizonte de tiempo definido.

\section{RESULTADOS Y DISCUSIÓN}

Los resultados de las funciones impulso respuesta para la industria minera se pueden observar en la gráfica 1 acorde al modelo VAR planteado en el vector $Y_{\text {minería }}{ }^{3}$. El primer resultado destacable es que cuando hay algún choque exógeno sobre la generación de $\mathrm{CO}_{2}$, este se refleja de forma positiva sobre los impuestos ambientales totales. En otras palabras, cuando algún evento produce un cambio en la emisión de este contaminante, los impuestos tienden a aumentar, dicho efecto se evidencia desde el segundo periodo (año). Por otro lado, la respuesta de la inversión total en ambiente y de las actividades de protección ambiental, sufren una leve caída estadística a valores no significativos en el primer periodo, pero luego del segundo periodo su respuesta es positiva y relevante en términos estadísticos. Por el contrario, cuando existe un cambio sobre los impuestos ambientales totales, la respuesta de todas las variables es negativa, pero no significativa estadísticamente. De todas formas, en términos analíticos el resultado puede representar que para el conjunto de variables consideradas cualquier cambio provocado en los impuestos suele disminuir los

La línea del medio en cada gráfica representa la función impulso respuesta, las líneas superior e inferior son sus límites de confianza al $95 \%$. 
niveles de $\mathrm{CO}_{2}$ para periodos subsiguientes, pero también afecta la inversión y las actividades relacionadas con la protección del ambiente en esta industria.

Las dos secciones siguientes del gráfico 1 detallan la respuesta de las variables consideradas en el modelo ante choques en la inversión total en medio ambiente y las actividades de protección ambiental. Para la primera de estas variables, la respuesta de la generación de $\mathrm{CO}_{2}$ resultó negativa, es decir, el nivel de $\mathrm{CO}_{2}$ decae cuando algún factor cambia la inversión total en medio ambiente. En el segundo caso, el efecto fue en parte contrario, es decir que hay una tendencia a aumentar la polución después del segundo periodo, cuando hay alguna afectación externa sobre las actividades de protección ambiental. Esto sucede a pesar de que en el primer periodo la función se comporta acorde a lo esperado, es decir, con una disminución en la cantidad de $\mathrm{CO}_{2}$. No obstante, este cambio es nulo en términos estadísticos, pues la función no logra que los límites de confianza sobrepasen el cero.

\section{Gráfico 1. Funciones impulso respuesta para el sector minero}
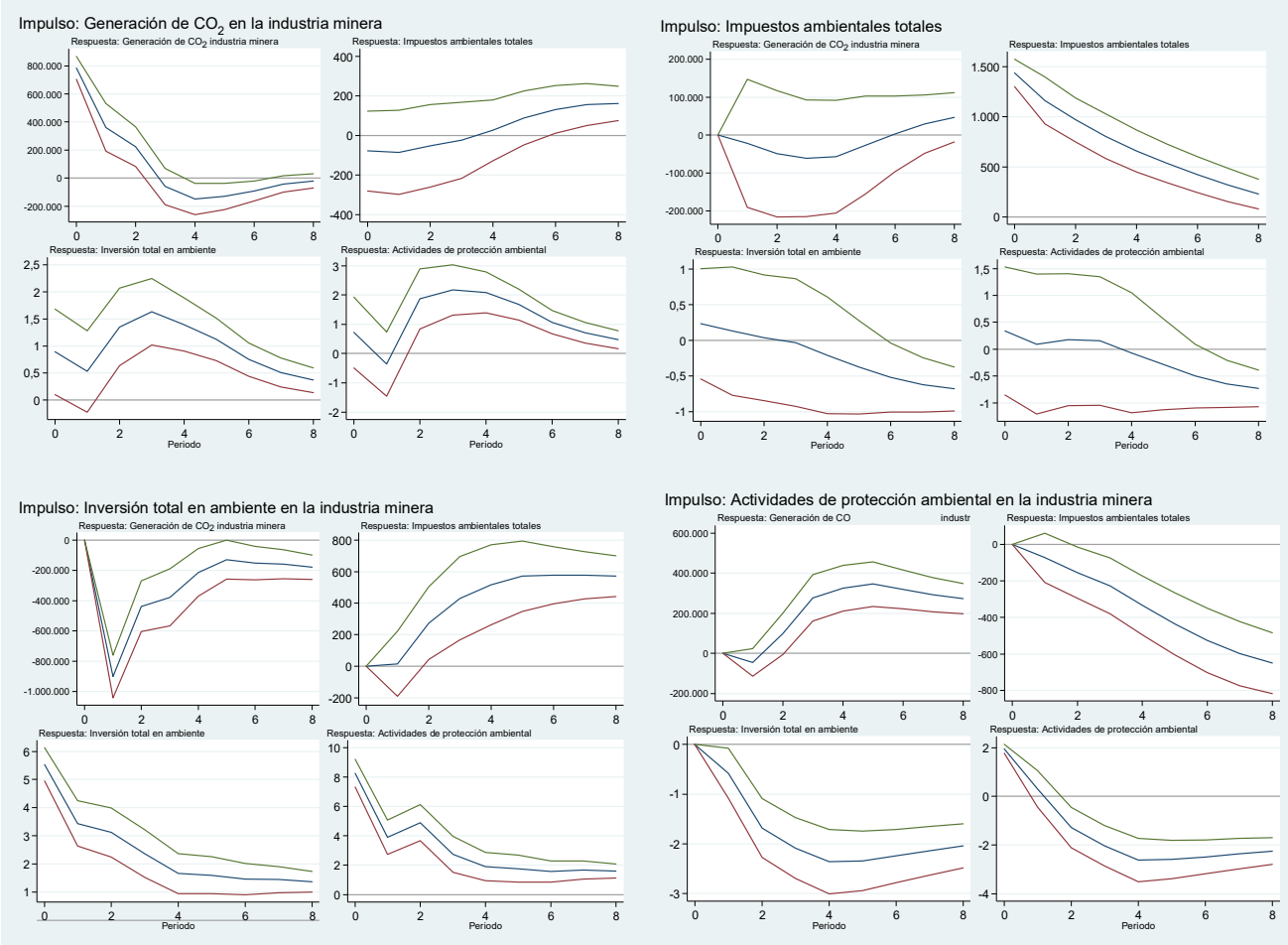

Fuente: elaboración propia en Stata 12.1. 
Ahora bien, al tener en cuenta que cada una de las variables influye sobre la generación de $\mathrm{CO}_{2}$, y para extender el análisis propuesto, en el gráfico 2 se muestra la función FEVD en donde se aprecia que, a pesar de que los impuestos no explican en ningún momento del tiempo el comportamiento de la emisión de este gas contaminante, sí lo hacen las inversiones totales del sector de minas. Además, tanto en el corto como en el largo plazo (a partir del periodo 8), los efectos de esta variable sobre la generación de $\mathrm{CO}_{2}$ se acercan al $50 \%$. Lo anterior sugiere que los impuestos no trabajan de forma directa sobre la reducción de la polución, pero la inversión en el ambiente sí lo hace, para contribuir con más de la mitad de dicho cambio.

\section{Gráfico 2. FEVD Industria minera}

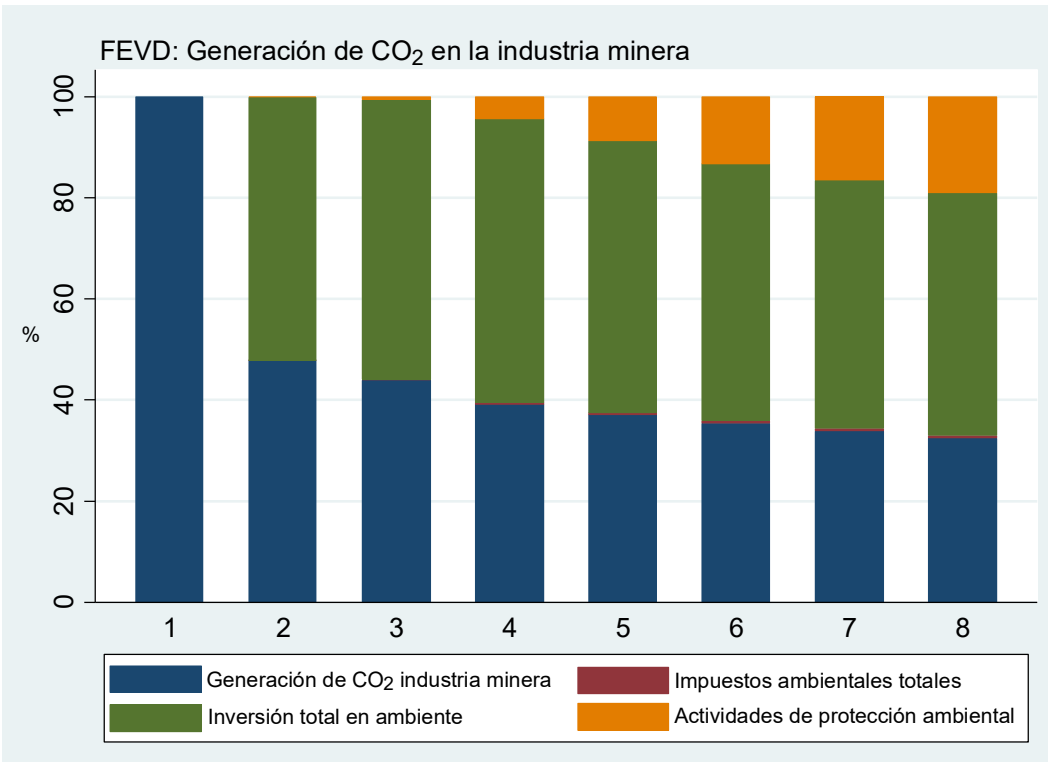

Fuente: elaboración propia en Stata 12.1.

Al continuar con el análisis, en la gráfica 3 se detallan las funciones impulso respuesta para el segundo modelo (vector $Y_{\text {Transporte), en el que se considera el }}$ sector energético de los países europeos. Acorde a la primera parte del gráfico 3, cualquier shock sobre los impuestos medioambientales, generaría una caída en la generación de $\mathrm{CO}_{2}$ a partir del primer periodo (año). No obstante, dicha reducción solo es significativa en términos estadísticos a partir del cuarto periodo, para luego volver a su senda de largo plazo. Esta respuesta poco sensible de la generación de $\mathrm{CO}_{2}$ ante los impuestos ambientales puede sugerir diferentes explicaciones. Una de ellas está definida por el sistema de tributación. Si los agentes tienen la capacidad 
adquisitiva de pagar el sobre costo generado, sus acciones no se verán reflejadas en una reducción de las emisiones e incluso puede evidenciarse un resultado contraproducente. Sumado a lo anterior, la respuesta del consumo final de energía para este sector reacciona de forma positiva cuando existen cambios en los impuestos. Sin embargo, este cambio no es significativo y tiene un punto de inflexión luego del primer periodo, para tornarse negativo y relevante en términos estadísticos desde el tercer periodo.

\section{Gráfico 3. Funciones impulso respuesta para el sector transporte}
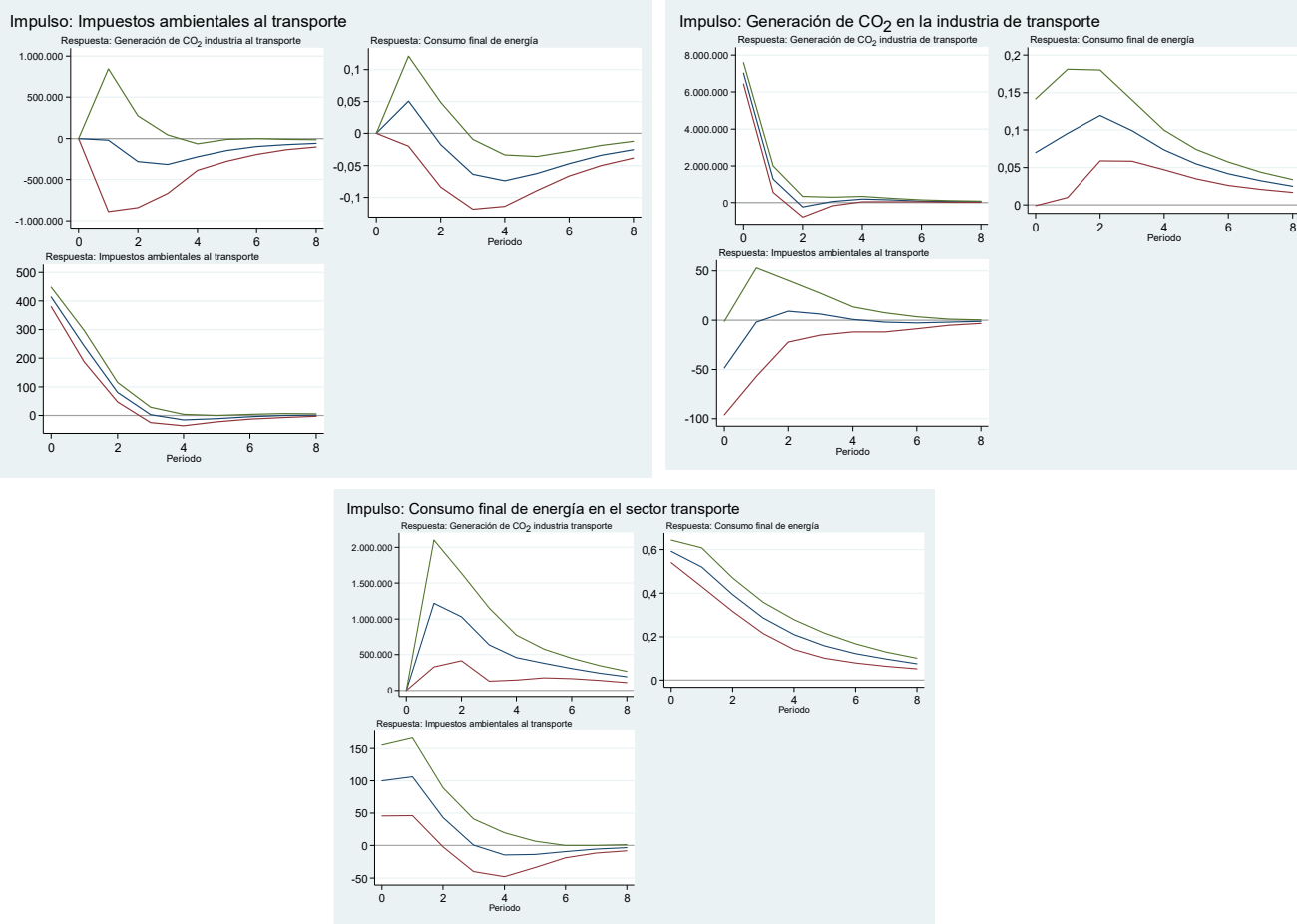

Fuente: elaboración propia en Stata 12.1.

De forma análoga, los choques por factores externos sobre la generación de $\mathrm{CO}_{2}$ en el sector transporte, generan una respuesta positiva, pero no significativa en los impuestos, mientras que en el consumo de energía el efecto primero mostró un incremento, para luego decaer. Por último, cualquier cambio sobre el consumo final de energía en este sector, genera una marcada respuesta positiva por parte de los impuestos y la generación de $\mathrm{CO}_{2}$, dicho incremento en ambas variables de forma paulatina vuelve a su senda de largo plazo entre el segundo y el tercer periodo. 
Ahora bien, con respecto a la función FEVD (gráfico 4), en el sector transporte la importancia relativa de las demás variables consideradas sobre la generación de $\mathrm{CO}_{2}$ fue casi nula. Se destaca que los impuestos no participaron de forma contundente ni siquiera en el largo plazo, de igual forma el consumo de energía tampoco participa. Por lo tanto, los factores inherentes a la producción de este gas contaminante prevalecen sobre las demás variables del modelo.

\section{Gráfico 4. FEVD sector transporte}

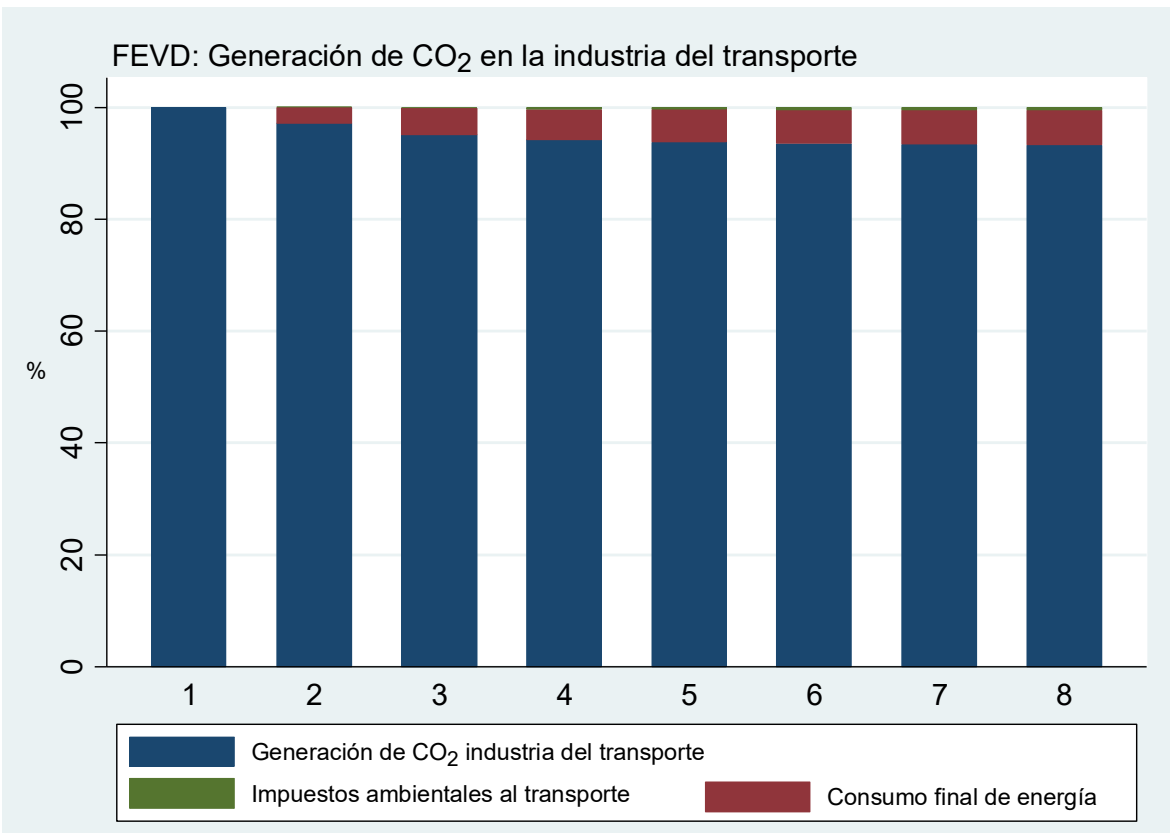

Fuente: elaboración propia en Stata 12.1.

\section{CONCLUSIONES}

De los resultados de las estimaciones del modelo PVAR y la herramienta FEVD se puede concluir que, para el caso de los treinta y un países de Europa, los impuestos ambientales no repercuten en la disminución de la emisión de $\mathrm{CO}_{2}$ para los sectores minero y de transporte.

Esto se podría explicar para el sector transporte, porque este es muy dependiente del petróleo y sus combustibles derivados, los cuales representan el 95 \% de su consumo final de energía (European Environment Agency, 2018), lo que podría mostrar la imposibilidad de sustituir en el corto plazo la energía fósil por otras alternativas 
más limpias. Las mejoras en la eficiencia en el consumo de combustibles fósiles han jugado su parte en esta explicación, para hacer que, frente a incrementos en la demanda de energía, las emisiones no respondan del todo a los impuestos. En respaldo de lo anterior, Greene et al. (2005) resaltan que los ahorros de combustible se deben a la adopción de tecnologías en lugar de a cambios en las ventas.

Es que después de alcanzar su punto máximo en 2007, el consumo de petróleo en el transporte (incluidos los bunkers marítimos) disminuyó en Europa de forma continua hasta 2013, cuando alcanzó un 11 \% por debajo de los niveles de 2007. Esto se debió a las mejoras en la eficiencia energética, los impactos de la recesión económica que causaron una disminución posterior en la demanda de transporte y un período de altos precios del petróleo después de 2010 como lo señala la European Environment Agency (2018), tendencia que muestra que los ciclos económicos y los precios también inciden en la demanda energética.

En contravía de los hallazgos propios, existe otra evidencia sobre los efectos positivos de los impuestos al $\mathrm{CO}_{2}$ en Europa. Andersen y Ekins (2010) muestran que los tributos ambientales contribuyeron para reducir las emisiones de gases de efecto invernadero en 7 \%. En esta misma línea argumental, Adolf (2013) muestra que en Alemania, el diseño de un paquete fiscal en 1999 resultó efectivo para mitigar la contaminación, lo que redujo las emisiones el 3 \% para el año 2010.

Lo que sí es hecho, es que el crecimiento de la producción primaria de la Unión Europea a partir de fuentes de energía renovables superó a la de todos los otros tipos de energía. Este crecimiento ha sido uniforme en términos relativos durante el período 2006-2016, con una pequeña caída en la producción en 2011. Durante dicho lapso de once años, los datos de Eurostat (2018), muestran que la producción de fuentes renovables aumentó un 66,5\%, para reemplazar la producción de otras fuentes de energía que se redujeron, como el gas natural (-41,2\%), el petróleo crudo $(-39,0 \%)$, los combustibles sólidos $(-30,8 \%)$ y la energía nuclear $(-15,2 \%)$.

En todos los esfuerzos por controlar las emisiones contaminantes, existen tareas pendientes como la que mencionan Andersen y Ekins (2010), con respecto al traslado de las emisiones a otros países y regiones que no cobran impuestos al carbono como ocurre con China y en otras economías emergentes.

Otra conclusión destacable es que los impuestos, aunque no actúan de forma directa, sí pueden lograr afectar otras variables, como el consumo de energía, para hacer que disminuya (después del tercer año como lo muestran los hallazgos para el sector transporte) o la cantidad de recursos destinados para actividades de protección del ambiente. Esto significa que la carga impositiva por sí sola no 
genera una reacción negativa en las emisiones, pero sí en el recaudo fiscal que puede ser utilizado para labores de saneamiento y prevención, y que como ocurre en el sector minero reducen las emisiones de $\mathrm{CO}_{2}$ por efecto de las inversiones totales en medio ambiente.

La respuesta de los impuestos puede que no sea inmediata, porque depende de otro tipo de circunstancias, como la voluntad política (Lamotte, 2013; Convery, Dunne y Joyce, 2013), las presiones de los diferentes sectores industriales, la dependencia por la energía de origen fósil, la falta de efectividad de los impuestos para llegar al óptimo social o la transmisión de un mensaje equivocado a los agentes.

De esto último, se desprende que algunos agentes consideren pagar por las emisiones de $\mathrm{CO}_{2}$ que generan, sin disminuir los contaminantes que liberan a la atmósfera, lo que podría mostrar un problema en el diseño del impuesto. Para Leicester (2013) esto pasa porque existen efectos sobre la conducta de los agentes económicos que son provocados por este tipo de medidas impositivas, en específico, por "motivaciones intrínsecas y extrínsecas". Manifiesta que las personas pueden tener una motivación intrínseca para no contaminar hasta que se impone un tributo (incentivo extrínseco), que puede hacer ver la contaminación como algo válido.

Para terminar, esta discusión es importante para Colombia, que recién empieza a aplicar impuestos a las emisiones de $\mathrm{CO}_{2}$ (a junio de 2017, según datos de la Dirección de Impuestos y Aduanas Nacionales (DIAN), tenía un recaudo de USD 159 millones y en donde, según Calderón et al. (2016), la intensidad del carbono en el sistema energético es baja en comparación con otros países de Latinoamérica, pese a que la tendencia está cambiando por el crecimiento de su economía y el potencial que tiene este país en el uso de tecnologías basadas en carbono.

\section{REFERENCIAS}

Adolf, Constanze (2013). De contrapartida financiera a oportunidad; de visión a concreción: la reforma fiscal medioambiental en Alemania y las oportunidades para la coordinación europea. En: Álvarez, Eloy y Larrea, Macarena (Coords.). Energía y tributación ambiental. Madrid: Marcial Pons, p. 81-98.

Andersen, Mikael y Ekins, Paul (2010). Carbon-Energy taxation: lessons from Europe. Nueva York: Oxford University Press, 344 p.

Barde, Jean (2002). Reformas fiscales ambientales: una revisión de la experiencia en países de la OCDE. En: Moreno, Graciela; Mendoza, Paola y Ávila, Sara (Comps.). Impuestos ambientales. 
Lecciones en países de la OCDE y experiencias en México. Ciudad de México: Instituto Nacional de Ecología, p. 13-32.

Baumol, William y Oates, Wallace (1988). The theory of environmental policy. Cambridge: Cambridge University Press, 299 p.

Bjørner, Thomas y Jensen, Henrik (2002). Energy taxes, voluntary agreements and investment subsidies - a micro-panel analysis of the effect on Danish industrial companies' energy demand. En: Resource and Energy Economics, vol. 24, n. ${ }^{\circ}$ 3, p. 229-249.

Bruvoll, Annegrete y Larsen, Bodil (2004). Greenhouse gas emissions in Norway: do carbon taxes work? En: Energy Policy, vol. 32, n. ${ }^{\circ} 4$, p. 493-505.

Buhl Pedersen, Preben; Ingerslev, Christina; Togeby, Mikael y Ahé, Gert (1998). Evaluation of the Danish Agreement System. Report plus Background Report. Copenhague: Danish Energy Agency, 33p.

Cagala, Tobias y Glogowsky, Ulrich (2014). XTVAR: Stata module to compute panel vector autoregression. https://bit.ly/3fmnlqV

Cagala, Tobias; Glogowsky, Ulrich; Grimm, Veronika y Rincke, Johannes (2015). Cooperation and Trustworthiness in Repeated Interaction. https://ideas.repec.org/p/zbw/vfsc 14/107597.html

Calderón, Silvia; Álvarez, Andrés; Loboguerrero, Ana; Arango, Santiago; Calvin, Katherine; Kober, Tom; Daenzer, Kathryn y Fisher-Vanden, Karen (2016). Achieving $\mathrm{CO}_{2}$ reductions in Colombia: effects of carbon taxes and abatement targets. En: Energy Economics, vol. 56, p. 575-586.

Convery, Frank; Dunne, Louise y Joyce, Deirdre (2013). El impuesto sobre el carbono en Irlanda. En: Álvarez, Eloy y Larrea, Macarena (Coords.). Energía y tributación ambiental. Madrid: Marcial Pons, p. 99-131.

Di Cosmo, Valeria y Hyland, Marie (2013). Carbon tax scenarios and their effects on the Irish energy sector. En: Energy Policy, vol. 59, p. 404-414.

Eurostat (2018). Production of primary energy decreased between 2006 and 2016. https://bit. ly/3hjaszn

European Environment Agency (2018). Progress of EU transport sector towards its environment and climate objectives. https://bit.ly/3hm6gPF

Gerlagh, Reyer y van der Zwaan, Bob (2006). Options and Instruments for a Deep Cut in $\mathrm{CO}_{2}$ Emissions: Carbon Dioxide Capture or Renewables, Taxes or Subsidies? En: The Energy Journal, vol. 27, n. 3 , p. 25-48.

González, Rodrigo y Hosoda, Eiji (2016). Environmental impact of aircraft emissions and aviation fuel tax in Japan. En: Journal of Air Transport Management, vol. 57, p. 234-240.

Greene, David; Patterson, Philip; Singh, Margaret y Li, Jia (2005). Feebates, rebates and gasguzzler taxes: a study of incentives for increased fuel economy. En: Energy Policy, vol. 33, n. ${ }^{\circ}$ 6, p. 757-775.

Hayashi, Yoshitsugu; Kato, Hirokazu y Teodoro, Rene (2001). A model system for the assessment of the effects of car and fuel green taxes on $\mathrm{CO} 2$ emissions. En: Transportation Research Part D: Transport and Environment, vol. 6, n. ${ }^{\circ}$ 2, p. 123-139. 
Helm, Dieter (2012). The Carbon Crunch: How we're getting climate change wrong -and how to fix it. Yale: Yale University Press, 304p.

Ito, Katsuya (2017). CO2 emissions, renewable and non-renewable energy consumption, and economic growth: evidence from panel data for developing countries. En: International Economics, vol. 151, p. 1-6.

Lamotte, Henri (2013). El impuesto sobre el carbono en Francia y el proyecto de 2009. En: Álvarez, Eloy y Larrea, Macarena (Coords.). Energía y tributación ambiental. Madrid: Marcial Pons, p. 67-80.

Leicester, Andrew (2013). Tributación medioambiental: principios económicos y experiencia en el Reino Unido. En: Álvarez, Eloy y Larrea, Macarena (Coords.). Energía y tributación ambiental. Madrid: Marcial Pons, p. 29-66.

Liang, Qiao-Mei; Fan, Ying y Wei, Yi-Ming. (2007). Carbon taxation policy in China: how to protect energy -and trade- intensive sectors? En: Journal of Policy Modeling, vol. 29, n. ${ }^{\circ}$ 2, p. 311-333.

Martínez-Alier, Joan y Roca-Jusmet, Jordi (2013). Economía ecológica y política ambiental. Ciudad de México: Fondo de Cultura Económica, 639p.

Nakata, Toshihiko y Lamont, Alan (2001). Analysis of the impacts of carbon taxes on energy systems in Japan. En: Energy Policy, vol. 29, n. ${ }^{\circ} 2$, p. 159-166.

OCDE - Organisation for Economic Cooperation and Development (2013). Effective Carbon Prices. https://bit.ly/3dWbPC2

Oueslati, Walid (2014). Environmental tax reform: short-term versus long-term macroeconomic effects. En: Journal of Macroeconomics, vol. 40, p. 190-201.

Parry, Ian y Small, Kenneth (2005). Does Britain or the United States Have the Right Gasoline Tax? En: The American Economic Review, vol. 95, n. ${ }^{\circ}$ 4, p. 1276 1289.

Pigou, Arthur (1920). The Economics of Welfare. Londres: Macmillan, 876 p.

Ryan, Lisa; Ferreira, Susana y Convery, Frank (2009). The impact of fiscal and other measures on new passenger car sales and $\mathrm{CO} 2$ emissions intensity: evidence from Europe. En: Energy Economics, vol. 31, n. ${ }^{\circ}$ 3, p. 365-374.

Tietenberg, Tom (1998). Disclosure Strategies for Pollution Control. En: Environmental and Resource Economics, vol. 11, p. 587-602.

EIA -U.S. Energy Information Administration (2016). Transportation sector energy consumption. https://bit.ly/3dXvrWv

Varian, Hal (2006). Microeconomía intermedia. Bogotá: Antoni Bosch, 781p.

Wang, Bing; Liu, Lei; Huang, Gordon; Li, Wei y Xie, Yulei (2018). Effects of carbon and environmental tax on power mix planning -A case study of Hebei Province, China. En: Energy, vol. 143, p. 645-657.

Wier, Mette; Birr-Pedersen, Katja; Jacobsen, Henrik y Klok, Jacob (2005). Are $\mathrm{CO}_{2}$ taxes regressive? Evidence from the Danish experience. En: Ecological Economics, vol. 52, n. ${ }^{2}$ 2, p. 239-251. 
Wissema, Wiepke y Dellink, Rob (2007). AGE analysis of the impact of a carbon energy tax on the Irish economy. En: Ecological Economics, vol. 61, n. ${ }^{\circ} 4$, p. 671-683.

Yábar, Ana (2016). El medio ambiente y su protección mediante instrumentos económicos. La experiencia internacional. En: García, María (Ed.). Instrumentos económicos y financieros para la gestión ambiental. Bogotá: Universidad Externado de Colombia, p. 19-37.

Zhang, Kun; Wang, Qian; Liang, Qiao-Mei y Chen, Hao (2016). A bibliometric analysis of research on carbon tax from 1989 to 2014. En: Renewable and Sustainable Energy Reviews, vol. 58, p. $297-310$. 


\section{ANEXOS}

Anexo 1. Tabla de correlaciones ${ }^{4}$

\begin{tabular}{|c|c|c|c|c|c|c|c|c|c|}
\hline \multicolumn{5}{|c|}{ Bélgica } & \multicolumn{5}{|c|}{ Bulgaria } \\
\hline & CT & EPE & EPA & ET & & CT & EPE & EPA & ET \\
\hline CT & 1 & & & & CT & 1 & & & \\
\hline EPE & 0,719 & 1 & & & EPE & 0,354 & 1 & & \\
\hline EPA & $-0,9369^{*}$ & $-0,5168$ & 1 & & EPA & $-0,4951$ & $-0,4036$ & 1 & \\
\hline ET & $-0,7822^{*}$ & $-0,3321$ & $0,8220^{*}$ & 1 & ET & 0,1664 & $-0,0104$ & 0,4428 & 1 \\
\hline \multicolumn{5}{|c|}{ República Checa } & \multicolumn{5}{|c|}{ Alemania } \\
\hline & CT & EPE & EPA & ET & & CT & EPE & EPA & ET \\
\hline CT & 1 & & & & CT & 1 & & & \\
\hline EPE & $-0,7053$ & 1 & & & EPE & 0,2722 & 1 & & \\
\hline EPA & 0,9956 & -1 & 1 & & EPA & $-0,3516$ & & 1 & \\
\hline ET & 0,5574 & 0,6601 & 0,9878 & 1 & ET & $-0,2533$ & $-0,5937$ & 0,3108 & 1 \\
\hline \multicolumn{5}{|c|}{ Italia } & \multicolumn{5}{|c|}{ Letonia } \\
\hline & CT & EPE & EPA & ET & & CT & EPE & EPA & ET \\
\hline CT & 1 & & & & CT & 1 & & & \\
\hline EPE & 0,6653 & 1 & & & EPE & 0,0189 & 1 & & \\
\hline EPA & $-0,9297^{*}$ & 0,7254 & 1 & & EPA & $-0,9441^{*}$ & 0,7598 & 1 & \\
\hline ET & $-0,8764^{*}$ & 0,1199 & $0,9203^{*}$ & 1 & ET & $-0,7418^{*}$ & 0,1015 & $0,9482^{*}$ & 1 \\
\hline \multicolumn{5}{|c|}{ Lituania } & \multicolumn{5}{|c|}{ Holanda } \\
\hline & CT & EPE & EPA & ET & & CT & EPE & EPA & ET \\
\hline CT & 1 & & & & $\mathrm{CT}$ & 1 & & & \\
\hline EPE & $-0,0046$ & 1 & & & EPE & 0,9847 & 1 & & \\
\hline EPA & 0,8706 & $-0,1846$ & 1 & & EPA & $-0,7306$ & & 1 & \\
\hline ET & 0,3765 & $-0,6106$ & 0,5423 & 1 & ET & 0,2854 & $-0,8132$ & 0,0105 & 1 \\
\hline
\end{tabular}

${ }_{4}$ Los resultados son de las correlaciones de Pairwise para los datos correspondientes al total de las industrias en ciertos países europeos. Las variables vienen definidas como sigue: Carbon dioxide-total - all NACE activitiestons (CT); Environmental protection expenditure-Euro per inhabitant (EPE); Total environmental protection activities-Million Euro (EPA); y Total environmental taxes-Million Euro (ET). Los asteriscos marcan la significancia de las correlaciones al $95 \%$ de confianza. Se presentan sólo los resultados para quince de los treinta y un países analizados, porque en algunos casos los datos faltantes impidieron el cálculo de las correlaciones. 


\begin{tabular}{|c|c|c|c|c|c|c|c|c|c|}
\hline \multicolumn{5}{|c|}{ Polonia } & \multicolumn{5}{|c|}{ Portugal } \\
\hline & CT & EPE & EPA & ET & & CT & EPE & EPA & ET \\
\hline $\mathrm{CT}$ & 1 & & & & CT & 1 & & & \\
\hline EPE & $-0,1309$ & 1 & & & EPE & $0,9677^{*}$ & 1 & & \\
\hline EPA & 0,5183 & 0,3258 & 1 & & EPA & -1 & -1 & 1 & \\
\hline ET & 0,4296 & $0,8248^{*}$ & $-0,1806$ & 1 & ET & $0,9091^{*}$ & $0,9534^{*}$ & -1 & 1 \\
\hline \multicolumn{5}{|c|}{ Serbia } & \multicolumn{5}{|c|}{ Eslovaquia } \\
\hline & CT & EPE & EPA & ET & & CT & EPE & EPA & ET \\
\hline $\mathrm{CT}$ & 1 & & & & CT & 1 & & & \\
\hline EPE & 0,3897 & 1 & & & EPE & 0,8615 & 1 & & \\
\hline EPA & -1 & -1 & 1 & & EPA & $-0,9589 *$ & $-0,8920^{*}$ & 1 & \\
\hline ET & 0,1037 & 0,9359* & 0,8831 & 1 & ET & $-0,1333$ & $-0,6526$ & $-0,4175$ & 1 \\
\hline \multicolumn{5}{|c|}{ Eslovenia } & \multicolumn{5}{|c|}{ España } \\
\hline & $\mathrm{CT}$ & EPE & EPA & ET & & CT & EPE & EPA & ET \\
\hline CT & 1 & & & & CT & 1 & & & \\
\hline EPE & $-0,0797$ & 1 & & & EPE & $0,9872^{*}$ & 1 & & \\
\hline EPA & 0,2886 & 0,6759 & 1 & & EPA & 0,82 & 0,9723 & 1 & \\
\hline ET & $-0,9414^{*}$ & 0,7398 & $-0,1958$ & 1 & ET & $-0,4308$ & $0,8344^{*}$ & $-0,6855$ & 1 \\
\hline \multicolumn{5}{|c|}{ Reino Unido } & & & & & \\
\hline & CT & EPE & EPA & ET & & & & & \\
\hline $\mathrm{CT}$ & 1 & & & & & & & & \\
\hline EPE & 0,9309* & 1 & & & & & & & \\
\hline EPA & $-0,3034$ & 0,9925 & 1 & & & & & & \\
\hline ET & $-0,3034$ & 0,2885 & 0,6063 & 1 & & & & & \\
\hline
\end{tabular}

Fuente: elaboración propia. 